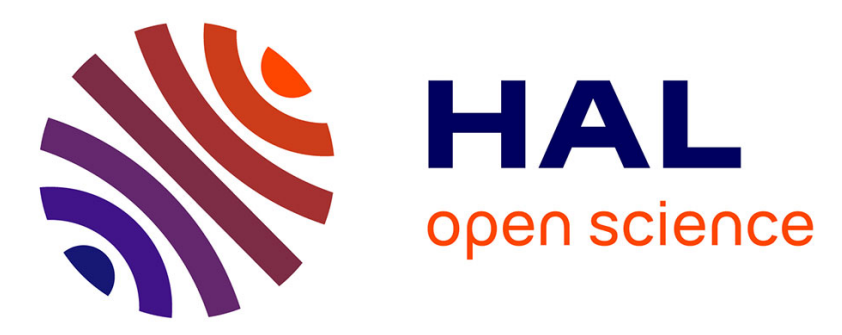

\title{
An Optimal Control Approach for EV Charging with Distribution Grid Ageing
}

Amar Prakash Azad, Olivier Beaude, Samson Lasaulce, Laurent Pfeiffer

\section{To cite this version:}

Amar Prakash Azad, Olivier Beaude, Samson Lasaulce, Laurent Pfeiffer. An Optimal Control Approach for EV Charging with Distribution Grid Ageing. 2013 First International Black Sea Conference on Communications and Networking (BlackSeaCom), Jul 2013, Batumi, Georgia. 10.1109/blackseacom.2013.6623411 . hal-01103809

\section{HAL Id: hal-01103809 \\ https://hal.science/hal-01103809}

Submitted on 15 Jan 2015

HAL is a multi-disciplinary open access archive for the deposit and dissemination of scientific research documents, whether they are published or not. The documents may come from teaching and research institutions in France or abroad, or from public or private research centers.
L'archive ouverte pluridisciplinaire HAL, est destinée au dépôt et à la diffusion de documents scientifiques de niveau recherche, publiés ou non, émanant des établissements d'enseignement et de recherche français ou étrangers, des laboratoires publics ou privés. 


\section{An Optimal Control Approach for EV Charging with Distribution Grid Ageing}

\author{
Amar Prakash Azad, Olivier Beaude, Samson Lasaulce \\ Laboratoire des Signaux et Systemes, CNRS, \\ Supelec, 3 rue Joliot-Curie 91191 Gif-sur-Yvette, France \\ Email: Amar.Azad;Olivier.Beaude;Samson.Lasaulce@lss.supelec.fr
}

\author{
Laurent Pfeiffer \\ INRIA Saclay and CMAP Polytechnique, \\ Palaiseau, France \\ Email: laurent.pfeiffer@polytechnique.edu
}

\begin{abstract}
In smart grids, the expected increase of electrical vehicle (EV) penetration will impose sizeable charging load, which can critically overburden the distribution network (DN) if the delivered power is non-pragmatically aggregated and induce significant impacts on various important existing grid assets. Among them, the residential distribution transformer is considered as one of the most important components in the grid. The ageing of the transformer is closely related to the temporal evolution of the hot-spot temperature (HST), which is induced by the operating load level history. We propose an optimal control approach to obtain a new EV charging algorithm: the novel aspect of this algorithm is that it takes inertial behavior of HST into account, which is the key parameter to capture the ageing. Though our formulation closely resembles to the linear quadratic control problem that includes costs induced from the state of the transformer and its present charging load, the natural constraints which are imposed to the instantaneous charging level (saturation constraints) induces intricate complicacy for the analytical solution. Thus, we follow the Pontryagin maximum principle approach to obtain the optimal charging policy and resort to numerical methods to compute the optimal charging trajectory. Numerical results allow us to evaluate and compare the performance of the proposed algorithm with various existing benchmark charging policies.
\end{abstract}

Keywords-distribution grid, electric vehicle (EV), smart grid, transformer ageing, dynamic control

\section{INTRODUCTION}

The automotive industry is heavily investing in plug-in hybrid electric vehicles (PHEVs) and fully electric vehicles (EVs) mainly in order to reduce the $\mathrm{CO}_{2}$ emissions and oil dependency of current automotive technology. The vehicle electrification $-13 \%$ of the vehicles will be full-electric and $23 \%$ will have an alternative powertrain by 2020 according to [1] - will have significant impacts on the power grid due to the increase in electricity consumption [2]. The charging of EVs has an impact on the distribution system because these vehicles consume a large amount of electrical energy and this demand of electrical energy can lead to large and undesirable peaks in the energy consumption [3].

One of the underlying critical issues is that the EV charging power is comparable to the maximum power corresponding to a typical (household) consumers subscription to an energy provider. In France, for instance, the former is typically about $3 \mathrm{~kW}$ at home while the latter is about $6 \mathrm{kVA}$, indicating the potential impact of charging EV. In this paper, this impact is considered as a linear combination of residential distribution transformer ageing and the distribution Joule losses [4]. As explained in [5], [6], optimizing a long-term cost such as

\footnotetext{
Author Olivier Beaude is associated with Renault SAS, France.
}

the residential transformers lifetime becomes an important concern in the presence of EVs. Indeed, transformers might have to operate in a regime where ageing is accelerated. Moreover, according to [7], about 70\% of transformer failures occurs because of this ageing and not because of fated events. Concerning Joule losses in the distribution network (DN), they represent the most important fraction of power losses in the whole energy network; according to [8], in France, two thirds of power losses is due to the DN.

In the recent literature, a number of scheduling schemes for optimal EV charging have been proposed, e.g. [9], where typically load reshaping is performed by peak shaving. In all these approaches instantaneous load level is accounted whereas the ageing cost requires a dynamical approach because of the historical load dependence. In [5], the ageing cost is studied for EV charging via simulations. To capture the temporal property of ageing cost, we follow the dynamic optimal control method, the Pontryagin maximum principle (PMP), to obtain the optimal EV charging schedule.

Compared with the existing literature, this paper makes the following main contributions: i) our model explicitly takes into account the dynamic property of hot-spot temperature (associated with thermal inertia) of the distribution transformer which depends on the load level history besides the instantaneous load; ii) we provide a formulation rooted in optimal control using Pontryagin maximum principle, which entails the optimal charging algorithm that minimizes the cost including physical and ageing components. To our knowledge, we are the first to consider the distribution network ageing cost with its dynamic behaviour and provide an optimal control approach to obtain the optimal EV charging policy.

The remainder of the paper is structured as follows: we introduce the model in Sec. II and propose the optimal control based policy using PMP approach in Sec. III. We then evaluate the performance of relevant EV charging algorithms via simulation in Sec. IV, and conclude in Sec. V.

\section{Proposed Modeling}

Consider a residential electrical DN in which a set of households and EVs are powered by an electric transformer in a community as in Fig. 1. The transformer (typically medium to low voltage) load has two components: the household load and the EV load. The electrical network operator is assumed to precisely predict the inelastic base load profile of the household and negotiates with the EVs to schedule their charging profiles over the $T$ time slots of length $\Delta T$ in future.

Increased persistent peak load due to additional PEVs could stress the residential transformer and degrade the transformer 


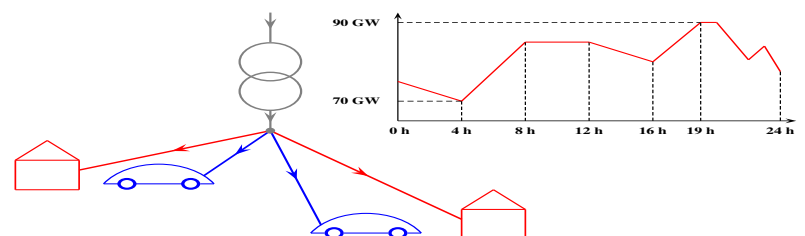

Fig. 1. Residential DN topology and load profile over a day (a typical case)

life. For example, if a transformer is overloaded during a time slot which leads to a HST larger than $98^{\circ} \mathrm{C}$, the IEEE standard level, then its life expectancy will decrease exponentially [7]. A PEVs penetration level as small as $10 \%$ could overload overhead distribution transformer [10] and increase distribution infrastructure costs. Transformers are among the most costly components in the medium/low voltage distribution infrastructure [2] and, therefore, transformer ageing is the key consideration for taking into account the impact of PEVs charging. The negative impact of PEVs can be minimized if smart charging methods can be deployed taking into account the distribution network cost [5]. In the following, we describe the detailed model of distribution transformer ageing and distribution network Joule losses.

\section{Physical Modeling of Distribution Network Cost}

Accurately estimating the impact of PEV charging on the distribution infrastructure requires to account both for the transformer life degradation as well as the physical losses in the topology dependent circuitary losses (Joule losses) [4].

Residential Transformer Ageing: Transformer ageing or deterioration results from insulation breakdown, which is directly related to the hot-spot temperature (HST) [11], [12], the most influential parameter (Annex G IEEE C57.91-95) ${ }^{1}$. The estimation of HST at time $t$ requires various other physical characteristics, e.g. ambient temperature, thermal capacity, loss ratio and thermal inertia [13]. Abstracting out the other factors, [13] suggested a model of HST as a non-linear function of load level, given by

$$
\theta_{t}=F_{t}^{\mathrm{HS}}\left(\mathbf{L}_{[t]}\right)
$$

where $\mathbf{L}_{[t]}=\left(L_{0}, L_{1}, \ldots, L_{t}\right)$ represents the sequence of total transformer load levels up to time $t$; the quantity $L_{t}$ is related to the charging power levels at time $t$. The measured time is normalized in the unit of time-slots. From [13, eq. (8)], [4] and with some approximation, we can express the temporal evolution (the inertial property) of the HST by the following simple and tractable form of difference equation:

$$
\theta_{t+1}=\alpha \theta_{t}+\beta_{t} L_{t}^{2}
$$

where $\alpha$ and $\beta_{t}$ depicts the thermal inertial constant and inertial load loss factor (refer [13] for more details). For a typical residential transformer, the operating values of $\alpha$ and $\beta_{t}$ lies in the range of $(0,1]$. Henceforth, we assume $\alpha>0$ and $\beta_{t}>0$ for the purpose of our analysis. A non-inertial version of (2) is considered in [4] in which HST was given as $\theta_{t}=\beta_{t} L_{t}^{2}$.

The transformer ageing acceleration factor (ageing in short) at time $t$ can be expressed as an exponential function of HST as $A_{t}=e^{a_{1} \theta_{t}+a_{2}}$. Typically, when operated close to nominal load, a Taylor series expansion (at $\theta_{t} \gg a_{1}^{-1}>0$ and $a_{2} \approx 0$ ) yields tractable form of the transformer ageing (see [12], [4] for more details)

\footnotetext{
${ }^{1}$ Typically, the HST also depends on ambient temperature history, which is an uncontrolled exogenous parameter (summer or winder, etc.) [12].
}

$$
A_{t}=1+a_{1} \theta_{t}+\frac{1}{2}\left(a_{1} \theta_{t}\right)^{2}=: f\left(\theta_{t}\right)
$$

Distribution Network Joule loss: We consider both the losses: i) in the distribution transformer; and ii) over the distribution lines between the transformer and the active electrical receivers; which include various electrical devices and the EVs (an example network of Fig.1). We assume that the transformer losses, e.g. iron losses, no-load losses, are negligible in magnitude as compared to line losses (for both fix frequency and voltage analysis). This assumption is also motivated by the fact that the transformer short circuit power is much more than the EV charging power. Moreover, the line losses are in proportion to the additional load of the EVs. Assuming constant current over the time-slot $t \in \mathcal{T}$, Joule losses (the mean square losses) are expressed as

$$
J\left(L_{t}\right)=R_{e q}\left(\sum_{i} L_{t}^{i}+L_{t}^{b}\right)^{2}
$$

where $R_{e q}=R_{\text {transfo }}+R_{\text {line }}$ is the equivalent resistance composed of transformer and line resistances (topology dependent), and $L_{t}=\sum_{i} L_{t}^{i}+L_{t}^{b}$, the aggregated transformer load.

\section{OPTIMAL EV CHARGING PROBLEM FORMULATION}

Consider the set of EVs, denoted by $\mathcal{I}=\{1, \cdots, I\}$, associated with the residential distribution network. Each EV can charge after it plugs in and needs to chafe a pre specified amount of electricity by its deadline. For instance, an EV may plug in for charging at 9:00 pm, specifying that it needs to be fully charged by 6:00 am, or reach at least 70\% state of charge (SOC) by then. Assume, during a time slot the charging rate of an EV is constant, denoted by $L_{t}^{i}$ (often called EV load level). Let $L_{t}^{b}$ denote the base load at time slot $t$. The key quantity to evaluate the impact of the charging policy on the distribution network is the total transformer load or consumed power. The $T+1$-dimensional sequence of the total load levels is $\mathbf{L}:=\left(L_{0}, L_{1}, \cdots, L_{T}\right)$ and charging rate at which current is delivered to the $i^{t h} \mathrm{EV}$ is denoted by $L^{i}=\left\{L_{0}^{i}, \cdots, L_{T}^{i}\right\}$ (we refer as $L_{(.)}^{i}$ at times).

Each EV $i \in \mathcal{I}$ can only charge after it plugs in(arrival) at $a_{i}$ and before its deadline(departure) $d_{i}$, i.e. $L_{t}^{i}=0$ if $t \notin$ $\left[a_{i}, d_{i}\right]$. We consider continuous level charging for a time slot during $t \in\left[a_{i}, d_{i}\right]$, i.e., an EV can be charged at any rate between 0 to maximum charging rate $L_{\max }$ (but constant $L_{t}^{i}$ in a time-slot) that is determined by the charger of the individual EV. For instance, for single-phase Level I charger $L_{\max }=1.4$ $\mathrm{kW}$ and for Level II charger $L_{\max }=3.3 \mathrm{~kW}$ [14]. Defining the charging profile upper bound $\bar{L}_{t}^{i}$ for a time slot $t \in \mathcal{T}$ as

$$
\bar{L}_{t}^{i}= \begin{cases}L_{\max } & \text { if } a_{i} \leq t \leq d_{i} \\ \underline{L} & \text { otherwise }\end{cases}
$$

where $\underline{L}$ denotes the minimum charging rate. Without loss of generality, we assume $\underline{L} \geq 0$ reflecting nonzero load offered by charging circuitry setup at no charging load. We consider same maximum charging rate for all EVs for simplicity (can be easily extended), i.e., $0 \leq \underline{L} \leq L_{t}^{i} \leq \bar{L}, t \in \mathcal{T}$, $i \in \mathcal{I}$. Based on the initial SOC and the final required SOC, a permissible optimal charging policy faces a constraint of minimum charging sum rate $\sum_{t \in \mathcal{T}} L_{t}^{i} \geq q_{i}$ for each $i \in \mathcal{I}$, where $q_{i}$ reflects the charging need to reach the final SOC. 
Consider the DN loss as a weighted combination of $A_{t}\left(\mathbf{L}_{t}\right)$, the ageing acceleration loss (with inertial component) and $J\left(L_{t}\right)$ the Joule losses, given by $g^{D N}(\mathbf{L})=$ $\sum_{t \in \mathcal{T}} \eta_{A} A_{t}\left(\mathbf{L}_{[t]}\right)+\eta_{J} J\left(L_{t}\right)$. Let $g_{i}^{E V}\left(L^{i}\right)=\eta_{E} \sum_{t \in \mathcal{T}} L_{t}^{i^{2}}$ be the individual local cost of the $i$ th EV, e.g., the impact of individual battery aging or the individual electricity fare. Let $p_{i}($.$) denote the pricing function which is an increasing$ function for the cost to the $i$ th $\mathrm{EV}$, then the cost to the $i$ th $\mathrm{EV}$ is $C_{i}(\mathbf{L})=p_{i}\left(g^{D N}(\mathbf{L})+g_{i}^{E V}\left(L^{i}\right)\right)$. Due to space limitation, we omit the details of the intermediate terms (refer [4] for details) and directly express the cost to the $i$ th EV in terms of HST and the load level as

$$
C_{i}(\mathbf{L})=\sum_{t \in \mathcal{T}}\left(\eta f\left(\theta_{t}\right)+\bar{\eta} L_{t}^{2}\right) .
$$

where we denote $\eta \in[0,1]$ as the weighting factor which balances the sensitivity of the DN cost with long term load $\theta_{t}$ vs the short term or immediate load $L_{t}$. We assumed equal $\mathrm{DN}$ cost sharing among EVs for (5), i.e., $p_{i}(x)=x / I$.

In what follows, we formulate the optimal charging problem for a single EV which optimizes the distribution network (DN) cost. Before proceeding, we emphasize that studying the single user charging problem than multi user is motivated due to many reasons. The foremost reason is the ease of exposition of the intricacy involved and the detailed insights of the optimal control problem (we provide in the next section). Secondly, we aim the scenarios where EVs are not imposed to follow the same charging policy but they have knowledge of each other's policy before its realization. Moreover, one possible way to implement our charging policy is by considering the base load and other EVs load as an exogenous load. Without any claim, we expect that a sensible asynchronous iterative algorithm will lead several EV's to achieve the optimal policy in a centralized manner by pre negotiation type mechanism.

\section{A. Dynamic Optimal Control Problem for EV Charging}

In order to obtain the optimal charging policy for the $i$ th $\mathrm{EV}$, we want to minimize the aggregate $\mathrm{DN}$ cost $C_{i}($.$) by con-$ trolling the charging load level $L_{t}^{i}$ during $t \in\{0, T\}$ subject to the minimum charging sumrate requirement and under the HST dynamics (2). We assume that the $i$ th $\mathrm{EV}$ has the knowledge of the exogenous load, i.e., the residential base load and the charging load of other EVs $\left(L_{t}^{b}+\sum_{j \neq i} L_{t}^{j}\right)$. Mathematically, the optimization problem can then be formulated as follows.

Problem 3.1: Find the control policy for $i$ th EV charging level $L_{\left({ }_{.}\right)}^{i}=\left(L_{0}^{i}, L_{1}^{i}, \cdots, L_{T}^{i}\right)$ which solves:

$\underset{L_{(\cdot)}^{i} \in \mathcal{L}}{\operatorname{minimize}} C_{i}(\mathbf{L})$, subject to $\left\{\begin{array}{l}\sum_{t \in \mathcal{T}} L_{t}^{i} \geq q_{i}, \\ \theta_{t+1}=\alpha \theta_{t}+\beta_{t} L_{t}{ }^{2},\end{array}\right.$

where $q_{i} \geq 0$ is dictated by the charging requirement, and $\theta_{t}$ represents the HST dynamics from (2). In the above, we denoted the admissible continuous charging load by $\mathcal{L}=[\underline{L}, \bar{L}]^{T}$.

Though problem 3.1 resembles to a standard LQ control in the first instance, any classical approach is inapplicable because of the bounded admissible control with the compounded complexity due to minimum charging constraint. This is a nonstandard dynamic optimization problem formulated to capture the thermal inertia. Therefore, we provide the explicit solution in the following where we use PMP. We note that even the single user case is complicated enough to obtain the structure of the optimal dynamic policy analytically. Moreover, the multi user problem formulation may require difference game approach, which is beyond the scope of this paper.

HST Evolution: To study the optimization problem, we need to characterize the HST evolution. From (2), the dynamics of the HST can be expressed as a difference equation

$$
\theta_{t+1}=\alpha \theta_{t}+\beta_{t} L_{t}^{2}, \text { where } \theta_{0}=\theta(0) .
$$

Recall that the HST initial state is positive ${ }^{2}$, i.e., $\theta(0) \geq 0$, and $\beta_{t} \geq 0$ for all $t \in \mathcal{T}$ (from the assumption).

Lemma 1: Consider the dynamics of the HST state $\theta_{t}$ given in (7). The HST dynamics, given by (7), is always positive, i.e. $\theta_{t}>0$ for all $t \in\{0, T\}$.

Proof: From (7), the HST state is given by the following:

$$
\theta_{t}=\theta_{0} \alpha^{t}+\sum_{k=1}^{t} \alpha^{t-k} \beta_{k-1} L_{k-1}^{2}, \forall t>1 .
$$

From the above equation $\theta_{t}>0$ for all $t \in\{0, T\}$ is directly established by noting the assumption $\beta_{t}>0$ and $\alpha>0$.

In the following, we obtain the optimal charging level $L_{(.)}$in each time slot. We assume $L_{(.)} \in \mathcal{L}$ as a piece-wise continuous function which remains fixed within a slot duration. Denote $\bar{Q}_{i}(T)=\bar{L} T$ (and $Q_{i}(T)=\underline{L} T$ ) be the charging sum rate when the $i$ th $\mathrm{EV}$ is charged at maximum (respectively minimum) charging rate for entire duration.

Optimal Charging Policy: Consider the problem of minimizing $C_{i}(\mathbf{L})$ with respect to $L_{(.)}^{i} \in \mathcal{L}$ subject to the constraint $\sum_{t \in \mathcal{T}} L_{t}^{i} \geq q_{i}$, then

i. If $\bar{Q}_{i}(T)<q_{i}$, then there is no feasible control policy.

ii. If $Q_{i}(T) \geq q_{i}$, then the optimal policy is $L_{t}^{i}=\underline{L}$.

iii. If $\bar{Q}_{i}(T)<q_{i} \leq \bar{Q}_{i}(T)$, then the optimal policy is computed by the Pontryagin maximum principle.

In the above, cases $\mathrm{i}$ and ii are trivial and follow directly from (6). We thus proceed with the case iii, working under the assumption $\underline{Q}_{i}(T)<q_{i} \leq \bar{Q}_{i}(T)$. Fixing any charging policy $L^{-i}$, we need to solve the following problem

$$
\begin{array}{r}
\underset{L^{i} \in \mathcal{L}}{\operatorname{minimize}} \sum_{t \in \mathcal{T}}\left(\eta f\left(\theta_{t}\right)+\bar{\eta} L_{t}^{2}\right)+\mu\left(q_{i}-\sum_{t \in \mathcal{T}} L_{t}^{i}\right), \\
\text { s.t. } \theta_{t+1}=\alpha \theta_{t}+\beta L_{t}^{2} .
\end{array}
$$

Note, the minimum charging constraint is incorporated in the objective function with $\mu \geq 0$ as a Lagrangian constant [15]. Observe, the Lagrangian constraint is active (from the assumption of part iii) which implies a positive $\mu$. To solve the above minimization, we use the maximum principle (in discrete time) [16, Chap 6]. Introduce the Hamiltonian

$$
\begin{aligned}
H_{t}\left(\theta_{t}, L_{t}^{i}, \lambda_{t+1}\right) & =\left(\eta f\left(\theta_{t}\right)+\bar{\eta}\left(L_{t}^{i}+L_{t}^{-i}\right)^{2}\right) \\
+ & \lambda_{t+1}\left(\alpha \theta_{t}+\beta_{t}\left(L_{t}^{i}+L_{t}^{-i}\right)^{2}\right)-\mu L_{t}^{i}
\end{aligned}
$$

where $\lambda_{(.)}$is the co-state variable and $L_{t}^{-i}=L_{t}^{b}+\sum_{j \neq i} L_{t}^{j}$ denotes the total complementing load at time $t$ to the $i$ th EV. Let $L^{i *}$ be the optimal control and $\theta^{*}$ be the associated state. By the Maximum principle, the Hamiltonian system is given by

\footnotetext{
${ }^{2}$ A positive initial HST state assumption is from the fact that in a typical window of observation, from evening to morning, the nominal residential load would be enough even if ambient temperature becomes negative [12], [11].
} 


$$
\left\{\begin{array}{l}
\theta_{t+1}^{*}=\frac{\partial}{\partial \lambda_{t+1}} H_{t}\left(\theta_{t}^{*}, L_{t}^{i^{*}}, \lambda_{t+1}\right), \\
\lambda_{t}=\frac{\partial}{\partial \theta_{t}} H_{t}\left(\theta_{t}^{*}, L_{t}^{i^{*}}, \lambda_{t+1}\right)
\end{array}\right.
$$

and the optimal control is such that for all $t \in \mathcal{T}$,

$$
L_{t}^{i^{*}}=\underset{L \in[\underline{L}, \bar{L}]}{\arg \min } H_{t}\left(\theta_{t}^{*}, L, \lambda_{t+1}\right) .
$$

Note that the first equation of (11) is nothing but rewriting the state variable of (7) from Hamiltonian. Recall, this represents the evolution of the HST state (lemma 1), which is a positive function. The second equation of (11) is the costate variable (often called as adjoint equation) that regulates the multiplier $\lambda_{t}$, which is in backward progression. Writing the explicit form of the second co-state variable, we have the difference equation

$$
\forall t \in \mathcal{T}, \lambda_{t}=\alpha \lambda_{t+1}+\eta f^{\prime}\left(\theta_{t}^{*}\right), \quad \lambda_{T+1}=0 .
$$

The terminal condition $\lambda_{T+1}=0$ in the above equation is because of the free terminal state under the optimal control [16]. A simple observation of (13) reveals that $\lambda_{t}$. is positive for $t<T$ (recall $\alpha \in(0,1]$ and $\left.\theta_{t}>0, \forall t\right)$.

Having noted the state and the co-state variable, we discuss the optimal control. The Hamiltonian $H($.$) is convex in L_{(.)}^{i}$, as a consequence of (10) and from the positivity of $\lambda_{t+1}$. In a discrete-time framework, the Hamiltonian is only stationary at $L^{i *}$, but since it is convex, it is also minimized by $L^{i *}$, and here the minimum is unique. In order to satisfy the constraints $\underline{L}<L_{(.)}^{i^{*}}<\bar{L}$, the optimal control takes the value either on the extremum or the interior point as per the following rule:

$$
L_{t}^{i^{*}}= \begin{cases}\underline{L}, & \text { if } \lambda_{t+1}>\frac{\mu}{2 \beta_{t}\left(\underline{L}+L_{t}^{-i}\right)}-\frac{\bar{\eta}}{\beta_{t}}, \\ \bar{L}, & \text { if } \lambda_{t+1}<\frac{\mu}{2 \beta_{t}\left(\bar{L}+L_{t}^{-i}\right)}-\frac{\bar{\eta}}{\beta_{t}}, \\ l_{t}^{0}, & \text { otherwise. }\end{cases}
$$

In the above equation, the interior optimal control is the solution to $\frac{\partial H(.)}{\partial L^{i}}=0$ which yields $l_{t}^{0}=\frac{\mu}{2\left(\bar{\eta}+\lambda_{t+1} \beta_{t}\right)}-L_{t}^{-i}$. The value of the Lagrange multiplier $\mu$ is linked with the identity (recall the active constraint)

$$
\sum_{t \in \mathcal{T}} L_{t}^{i^{*}}=q_{i}
$$

By assumption, $L_{t}^{i *}$ cannot take only one solution, either $\bar{L}$ or $\underline{L}$, for the entire duration $T$. So, it must switch if it takes either of the two. However, an interior optimal policy $l_{t}^{0}$ may retain for the entire duration.

The problem cannot be solved analytically. Further, in this setting, it is even impossible to obtain some apriori information on the structure of the optimal control, such as the number of switches from one to another among the three possible behaviours (given by (14)). This is because it involves the interdependent co-state equations which further depend on the dynamic complementing load $L_{t}^{-i}$. Thus, we proceed to the well known numerical approach, the shooting method [17], to compute the optimal charging control from the above set of equations. The key point is to solve the backward and forward co-state set of equations numerically. We skip the mathematical details due to space limitation, rather we provide the numerical results in the next section with brief description.

One of the key relevance of the optimal control approach described above is to show the methodology for the dynamic

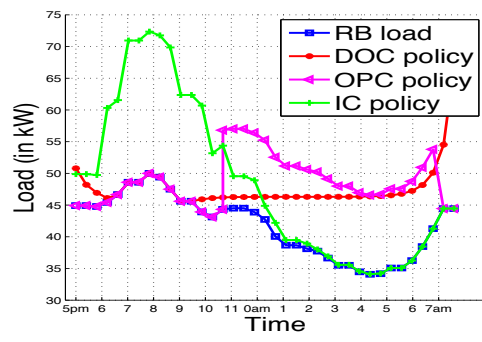

(a) Aggregate transformer load

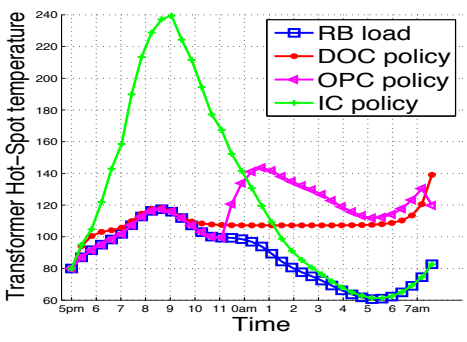

(b) HotSpot Temperature
Fig. 2. Performance comparison of charging policies over a typical day

EV charging problem which is the core technique to capture the inertial component of the DN cost. Though, obtaining analytical solution appears difficult for the general scenario, obtaining optimal charging control in special cases may be feasible, e.g., the symmetric charging control for multi EV in a centralized system. Due to space limitation, we skip detailed discussion about the special case, but we mention that our approach can be easily extended to such special cases by simply considering the vectorial form. Indeed, we present the multi EV charging performance in the next section using numerical method.

\section{Performance Evaluation}

We consider an urban distribution network with a 50kVA residential transformer using the ANSI/IEEE linearized Clause 7 [13]. The night time between 5 pm to 7 am of the next day is observed with a time step of 3 minutes. For the residential base load $L^{b}$, French data of 11-12th october 2011 night (available on $\mathrm{RTE}^{3}$ ) is scaled down to a district consumption level. It is assumed that the transformer's base load corresponds to $100 \%$ of its full $\operatorname{load}^{4}$. This relates to a district of 15 to 20 households. Initial transformer temperature of $80^{\circ} \mathrm{C}$ is used for the simulations. It is further assumed that each EV's charging requirement is approximately $20 \mathrm{kWh}$ and we consider $1-5 \mathrm{EVs}$ for evaluation (a penetration rate of $5-25 \%$ ). There are about 1 million such urban transformers in France, each costing about $5 k \$ s$ with 40 years of standard lifetime (according to IEC). The inertial parameters used for evaluations are $\alpha=0.95$ and $\beta=0.05$ (from [18]). Following are the charging policies adopted for performance evaluation, in which:

i) Off peak charging (OPC) policy: charging of EVs is triggered during the off peak hours with a constant charing rate $^{5}$, typically linked with the load valley.

ii) Immediate charging (IC) policy: charging of EVs begins as soon as they arrive and plug-in (the arrival time is normally distributed with mean at $8 \mathrm{pm}$ and standard deviation of 1 hour) at a slow charging rate $(P=3 k W)$.

iii) Dynamic optimal charging (DOC) policy: the proposed optimal charging policy which includes the key inertial property of transformer ageing.

In order to calculate the DOC policy, we use a shooting method [17]. The key idea is to determine $\lambda_{0}$ and $\mu$ such that $\lambda$ is a solution of (13) and the charging need constraint is simultaneously verified. Given an initial guess of $\left(\lambda_{0}, \mu\right)$, the dynamical system of (11) is solved using the the optimal control from (14). The initial guess is then iterated with

\footnotetext{
${ }^{3}$ The french electric TSO website: http://clients.rte-france.com/.

${ }^{4}$ In [5], the $25 \mathrm{kVA}$ transformer considered was subject to a peak load of $25.97 \mathrm{kVA}$.

${ }^{5}$ In France, Off peak charging is operational even for elastic residential appliances, e.g. water heaters.
} 
a descent method to satisfy (15) and to yield $\lambda_{T+1}=0$. Algorithm 1 summarizes this approach.

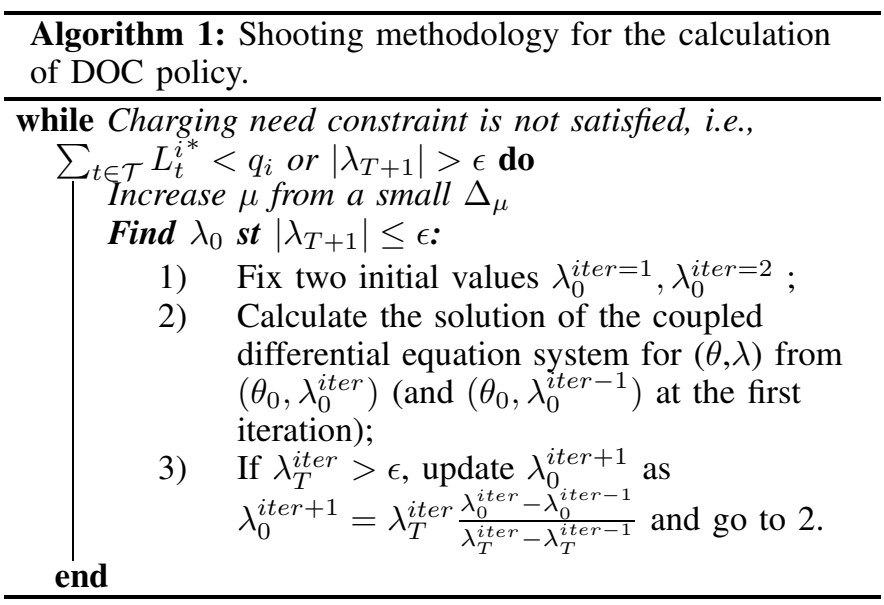

The performance evaluation comparison of different charging policies is depicted in Fig. 2 with $5 \mathrm{EV}$. For the DOC policy, all EVs are considered arriving at the same time. The load in Fig 2(a) refers to the transformer aggregated load which is composed of both the EV load and the existing residential base load. The base load is also explicitly depicted in Fig. 2(a) as RB Load.

In Fig 2(a), it appears that the DOC uses the "night valley" with a slightly delayed charging which is because of the ageing factor, the key aspect of DOC. This delay is mainly because of the thermal inertia of the HST. This induces the charging load towards the end which is reflected by the significant increase of both load and HS temperature (in Fig. 2(b)) to the morning time $7 \mathrm{pm}$ when all EV must depart. We also emphasize that the particular deep of base load in the night valley doesn't allow DOC to show significant improvement over OPC. However, in favorable situation we can expect DOC to perform better.

The final metric to measure the benefit of the charging policies is the transformer's lifetime, which is shown in Table IV. Observe, the lifetime decreases with increasing number of EVs (depreciated up to 23 years). Notice that both DOC and OPC policies show improvement as compared to IC. At the same time, the performance of DOC policy and OPC policy closely follow each other, which is because of their ability to exploit the night valley. Again, these policies may be significantly distinguished with other types of load profiles e.g., during the day.

TABLE I. TRANSFORMER LIFETIME IN YEARS
\begin{tabular}{|c|c|c|c|}
\hline (LIFETIME WITHOUT EV - 40 YRS) \\
\hline 1 & 37 & 36.5 & 35 \\
\hline 3 & 31 & 29.5 & 29 \\
\hline 5 & 25.5 & 23.5 & 17 \\
\hline
\end{tabular}

\section{CONCLUDING REMARKS}

In this paper, we proposed an optimal control approach for scheduling EV charging by taking into account the distribution network stress with the key inertial component of the hotspot temperature. Using Pontryagin maximum principle, we obtained the optimal charging policy, which is unique, and evaluated its performance. For special cases, the optimal charging policy can be expressed analytically. For instance, when Joule losses are dominant, the obtained solution is a valley-filling solution over the exogeneous demand profile. But, in general, when the transformer ageing is non-negligible or even dominant, it is seen that we have to resort to a numerical technique (namely, a shooting method) to determine the optimal policy. For the case where the transformer ageing is dominant, our proposed optimal policy shows significant benefit in terms of transformer lifetime via simulations. Note that we have adopted a single agent optimal control approach whereas the number of EVs is generally greater than one. In fact, the proposed approach can be seen as a way of determining in a centralized manner the set of charging policies which should be used by a given set of EVs or a single-user approach of a multiuser problem. However, it is of interest in devising decentralized charging policies for the problem under investigation in this paper. This constitutes a relevant extension of this work.

\section{REFERENCES}

[1] Eurotaxglass. Residual value trends for alternative powertrain vehicles in europe residual value and volume forecasts for the big 5 markets in europe (germany, france, italy, spain, uk). Technical report, 2008.

[2] L. P. Fernández, T.G.S. Romn, R. Cossent, C. M. Domingo, and P. Fras. Assessment of the impact of plug-in electric vehicles on distribution networks. IEEE Transactions on Power Systems, 26(1):206-213, 2011.

[3] K. Clement-Nyns, E. Haesen, and J. Driesen. The impact of charging plug-in hybrid electric vehicles on a residential distribution grid. IEEE Transactions on Power Systems, 25(1):371-380, 2010.

[4] O. Beaude, S. Lasaulce, and M. Hennebel. Charging games in networks of electrical vehicles. In IEEE NetGCooP, pages 96-103, 2012.

[5] Q Gong, S Midlam-Mohler, V Marano, and G Rizzoni. Study of PEV charging on residential distribution transformer life. IEEE Transactions on Smart Grid, 2011.

[6] D. K. McBee and G. M. Simoes. Reducing distribution transformer losses through the use of smart grid monitoring. North American Power Symposium (NAPS), pages 1-6, 2009.

[7] X. Zhang, E. Gockenbach, V. Wasserberg, and H. Borsi. Estimation of the lifetime of the electrical components in distribution networks. IEEE Transactions On Power Delivery, 22:515-522, 2007.

[8] E. Dyevre, P. Bornard, J. Conil-Lacoste, R. Durdilly, P. Fontaine, J. Keppler, T. Pons, E. Rodriguez, and J.L.R. Sanchez. Systems for covering power losses in public electric power network. Technical report, French Energy Regulatory Commission (CRE), 2010.

[9] L. Gan, U. Topcu, and S. Low. Optimal decentralized protocol for electric vehicle charging. In IEEE CDC-ECC, pages 5798-5804, 2011.

[10] S. Shafiee, M. Fotuhi-Firuzabad, and M. Rastegar. Investigating the impacts of plug-in hybrid electric vehicles on power distribution systems. Smart Grid, IEEE Transactions on, PP(99):1-10, 2013.

[11] Guide for loading mineral-oil-immersed transformers. IEEE Std. C57.91-1995, 1995.

[12] IEC-Loading guide for Oil-Immersed Power Transformers. No.- 60354.

[13] L.J. Rivera and D.J. Tylavsky. Acceptability of four transformer top-oil thermal models: Pt. 1: Defining metrics. IEEE Transactions On Power Delivery, 23:860-865, 2008.

[14] N. Leemput, J. Van Roy, F. Geth, P. Tant, and J. Driesen. Comparative analysis of coordination strategies for electric vehicles. In IEEE PES Innovative Smart Grid Technologies Conference, December 2011.

[15] S. Boyd and L. Vandenberghe. Convex Optimization. Cambridge University Press, 2004.

[16] A. P. Sage and C. C. White. Optimum systems control. Prentice-Hall, 1977.

[17] Arthur E. Bryson, Jr. and Yu Chi Ho. Applied optimal control. Hemisphere Publishing Corp. Washington, D. C., 1975.

[18] L. J. Rivera and D. J. Tylavsky. Acceptability of four transformer topoil thermal models: Pt. 2: Comparing metrics. IEEE Transactions On Power Delivery, 23:866-872, 2008. 